九州大学学術情報リポジトリ

Kyushu University Institutional Repository

\title{
Three-dimensional Free Surface Flows Modeled by Lattice Boltzmann Method: A Comparison with Experimental Data
}

Mohd, Nik

Interdisicplinary Graduate School of Engineering Sciences (IGSES), Kyushu University

Kamra, Mohamed M.

Interdisicplinary Graduate School of Engineering Sciences (IGSES), Kyushu University

Sueyoshi, Makoto

Research Institute for Applied Mechanics (RIAM), Kyushu University

$\mathrm{Hu}$, Changhong

Research Institute for Applied Mechanics (RIAM), Kyushu University

https://doi.org/10.5109/1808450

出版情報: Evergreen. 4 (1)，pp.29-35，2017-03. Green Asia Education Center バージョン：

権利関係 : 


\title{
Three-dimensional Free Surface Flows Modeled by Lattice Boltzmann Method: A Comparison with Experimental Data
}

\author{
Nik Mohd ${ }^{1}$, Mohamed M. Kamra ${ }^{1}$, Makoto Sueyoshi ${ }^{2}$, Changhong $\mathrm{Hu}^{2, *}$ \\ ${ }^{1}$ Interdisicplinary Graduate School of Engineering Sciences (IGSES), Kyushu University, Japan \\ ${ }^{2}$ Research Institute for Applied Mechanics (RIAM), Kyushu University, Japan \\ *Author to whom correspondence should be addressed, \\ E-mail: hu@riam.kyushu-u.ac.jp
}

(Received January 10, 2016; accepted March 15, 2017).

\begin{abstract}
Three-dimensional numerical simulations of strongly nonlinear free surface flows are performed by lattice Boltzmann method (LBM), which features a number of performance-related advantages, particularly concerning data locality and parallel computing. A Multi-Passage-Interface (MPI) multicore processors parallelized free surface LBM solver is applied for the present three-dimensional numerical simulations. A Smagorinsky LES turbulent model serves to capture the small-scale turbulent structures of the flow. Experiments on dam breaking from previous articles are used to compare and verify two-dimensional (2D) and three-dimensional (3D) LBM model. A new experimental setup is also developed in order to observe the three-dimensionality effect. The findings demonstrated that the free surface LBM simulation agrees well with the experiments.
\end{abstract}

Keywords: Free surface flow; lattice Boltzmann method; MPI

\section{Introduction}

Strongly nonlinear free surface flow has been a significant subject of study for the applications of ocean and coastal engineering which include wave breaker design, prediction of dam breaking flow and tsunami flow $^{1,2)}$.

Many previous studies have also investigated on the evaluation of kinematics wave and impact force on coastal engineering. Research on dam breaking research has contributed significantly towards the understanding of the behavior of complex nonlinear free surface flow. Studies on dam breaking flow can be broadly categorized as experimental and numerical modeling with the first studies analyzing dam breaking flow dated back in the 19th century3).

Some of the early experimental measurement studies conducted include a study which performed a series of tests and produced a comprehensive set of data on kinematics of two- dimensional (2D) as well as threedimensional (3D) dam break flow over an initially dry horizontal bed ${ }^{4}$. At present, the study has been used widely as a benchmark for dam-breaking studies. Other experimental studies include an experiment on dam breaking problem which served as a validation for the authors' own numerical methods ${ }^{5}$. A more recent experimental study paid special attention to the kinematic and dynamics impact of the downstream wave on the flat vertical walls ${ }^{3)}$. In the previous studies cited above, none of them studied the effect of splash after-impact although the splash effect is an important indicator to measure wave impact. Furthermore, the lack of detailed observation on the effect of 3D in these studies becomes the motivation for the current study.

Studies on numerical modeling ${ }^{6,7)}$ have focused on the validation of their numerical scheme using an experimental work performed at the Maritime Research Institute of the Netherlands (MARIN) that provided a description of dam breaking wave kinematics as well as the data of a wave impact on a solid vertical wall downstream from the dam. Another study developed two methods based on the Constrained Interpolation Profile (CIP)-Cartesian grid method and Moving Particle Semiimplicit (MPS) based particles in order to investigate the kinematics flow of free surface ${ }^{5)}$. Using this study's experimental platform, another study investigated the phenomenon of free surface flow impacting on elastic structures whereby a free surface algorithm with coupled Finite Different Method (FDM)-Finite Element Method (FEM) method was developed ${ }^{8}$. Recently, a study investigated the gate motion effect on dam breaking flow which discovered a significant gate motion effect using a (CIP)-Cartesian grid based model employed with the free surface boundary condition to deal with the water-air-gate interactions ${ }^{9)}$

The Lattice Boltzmann method (LBM) provides an alternative way to solve fluid flows, particularly due to its efficient approach for solving a variety of complex fluid dynamics problems, such as in the field of multiphysics ${ }^{10)}$. Recently, it has become an alternative to conventional 
methods such as FDM and Finite Volume Method (FVM) on the basis of the Navier-Stokes equations ${ }^{11,12)}$ especially over its advantages in dealing with complex boundaries, incorporating of microscopic interactions, flexible reproduction of interface between multiple phases, and parallelization of the algorithm ${ }^{13)}$.

The objective of this study is to investigate the effect of three-dimensionality free surface flow. Firstly, we aimed to validate the numerical model LBM code based on a 2D in-house code ${ }^{14)}$ and 3D based on LBM open-source ${ }^{15}$, and secondly, we aimed to develop a 3D modeling based on LBM open-source ${ }^{15}$ including the splash effect.

\section{Computational Model}

\subsection{Lattice Boltzmann Method (LBM)}

The particle distribution function $f(t, x, \xi)$ is the main variable of microscopic approaches in which the normalized probability encounter a particle at position $\boldsymbol{x}$ at time $t$ with velocity, $\xi$. The Boltzmann equation describes the evolution of these distribution function by

$$
\frac{D f}{D t}=\frac{\partial f(t, \boldsymbol{x}, \boldsymbol{\xi})}{\partial t}+\xi \cdot \frac{\partial f(t, \boldsymbol{x}, \boldsymbol{\xi})}{\partial x}=\Omega
$$

The equation on the left-side is an expression of the advection term, while the collision term operator, $\Omega$ describes in interactions of particles on the microscopic scale. To acquire a model with lessened computational $\operatorname{cost}^{16)}$, the Boltzmann equation is discretized in the velocity space, $\xi$.

Here, the D3Q19 model ${ }^{17)}$ is used, which presents the following ten discretized microscopic particle velocity $e_{i}=$ $\{0,0,0\},\{ \pm c, 0,0\},\{0, \pm c, 0\},\{0,0, \pm c\},\{ \pm c, \pm c, 0\}$, $\{ \pm c, 0, \pm c\},\{0, \pm c, \pm c\}, i=0, \ldots, 18$ where a constant velocity $c$ affects the speed of sound $c_{s}=c / \sqrt{3}$. The following results of discrete Boltzmann equations

$$
\frac{D f_{i}}{D t}=\frac{\partial f_{i}(t, \boldsymbol{x}, \boldsymbol{\xi})}{\partial t}+\xi_{i} \cdot \frac{\partial f_{i}(t, \boldsymbol{x}, \boldsymbol{\xi})}{\partial x}=\Omega_{i}
$$

has to be discretized in space and time. This is accomplished by using a standard finite different discretization, in space and time, on a grid with $c=$ $\Delta x / \Delta t$ (grid spacing, and $\Delta x$ time step, $\Delta t$ ), which results to the following lattice Boltzmann equation

$$
f_{i}\left(t+\Delta t, \boldsymbol{x}+\boldsymbol{e}_{\boldsymbol{i}} \Delta t\right)-f_{i}(t, x)=\Omega_{i}
$$

Lastly, eq. (3) may be divided into a non-linear collision step and non-linear propagation step $f_{i}(t, x)+\Omega_{i}$ and $f_{i}\left(t+\Delta t, \boldsymbol{x}+\boldsymbol{e}_{\boldsymbol{i}} \Delta t\right)=\bar{f}_{i}(t, x)$

The solution of the lattice Boltzmann equation Eq.(3) has been well-accepted to satisfy the incompressible Navier-Stokes equation up to errors of $\mathcal{O}\left(\Delta x^{2}\right)$ and $\mathcal{O}\left(M a^{2}\right)$. The relationship between the macroscopic values for the hydrodynamics pressure and macroscopic fluid density $\rho$ and velocity $u$ as following:

$$
\rho(\boldsymbol{x}, t)=\sum_{i=0}^{18} f_{i}(\boldsymbol{x}, t)
$$

$$
u(\boldsymbol{x}, t)=\frac{1}{\rho} \sum_{i=0}^{18} e_{i} f_{i}(\boldsymbol{x}, t)
$$

$$
p(\boldsymbol{x}, t)=c_{s}^{2} \rho(\boldsymbol{x}, t)=c_{s}^{2} \sum_{i=0}^{18} f_{i}(\boldsymbol{x}, t)
$$

\subsection{Treatment of free surface}

In the current study, the algorithm proposed in the previous studies ${ }^{18,19)}$ which uses the volume of fluid (VOF) to track the interface motion is used to capture free surface flow. The tracking of the movement of the fluid interface is made through the calculation of the mass that is comprised in each cell. Two additional values, the mass, $m$ and the fluid fraction, $\varepsilon$ to be kept in each cell and their relationship as follow

$$
\varepsilon=m / \rho
$$

Similar with VOF approach as mentioned from literature ${ }^{19)}$, the tracking of the interface motion is made by computing the fluxes between cell. For interface cells, a direct calculation of the mass exchange is made from the stream step of the LBM. For an interface cell at $x$, the mass balance with a neighbor at $x+\Delta t e_{i}$ is specified by

$$
\begin{aligned}
& \Delta m_{i} \\
& =\left\{\begin{array}{c}
0 \\
f_{i}\left(x+\Delta t e_{i}, t\right)-f_{i}(x, t) \\
0.5 *\left[\epsilon\left(x+\Delta t e_{i}, t\right)+\varepsilon(x, t)\right] \cdot\left[f_{i}\left(x+\Delta t e_{i}, t\right)-f_{i}(x, t)\right]
\end{array}\right.
\end{aligned}
$$

In order to represent the behavior of the free surface, a special boundary condition is used. However, since the gas phase is not calculated, no PDFs are available for the gas cells. Therefore, after the stream step, the interface cells do not have full set of PDFs. The missing PDFs are 
rebuilt as suggested in literature ${ }^{18,19)}$

$f_{\tilde{\imath}}^{\prime}(\boldsymbol{x}, t+\Delta t)=f_{i}^{e q}\left(\rho_{A}, \boldsymbol{u}\right)+f_{\tilde{\imath}}^{e q}\left(\rho_{A}, \boldsymbol{u}\right)-f_{\tilde{\imath}}(\boldsymbol{x}, t)$

Velocity of respective cell at certain position $(\boldsymbol{x})$ id defined as $\boldsymbol{u}$, and time, $t$ which is referring to Eq.(6). Using the density of the equilibrium distribution function as $\rho_{A}$, the pressure at the atmosphere which is acting at the fluid interface is introduced. The complete set of PDFs for interface cell can be calculated by applying the above equation, Eq. (10).

\subsection{Turbulent Smagorinsky LES}

The strongly violent free surface flows in the high Reynolds number flow regime, where turbulent flows contribute to the general strong flow pattern is investigated in the ocean and coastal engineering applications $^{14)}$. For capturing such turbulent flow structures at the sub-grid scale, a Large Eddy Simulation (LES) turbulent model is combined with the $\mathrm{LBM}^{21)}$. In LES models, the subgrid-scale eddies are modeled by a subgrid spatial filter and the large spatial-scale turbulent eddy structures which transport the majority of the energy is solved directly. The Smagorinsky model which determines the strain rate tensor with Smagorinsky constant and strain rate tensor can be computed locally from the LBM. The LBM implementation of the Smagorinsky model can be referred in other studies ${ }^{22-23)}$ not mentioned here.

\subsection{Numerical implementation}

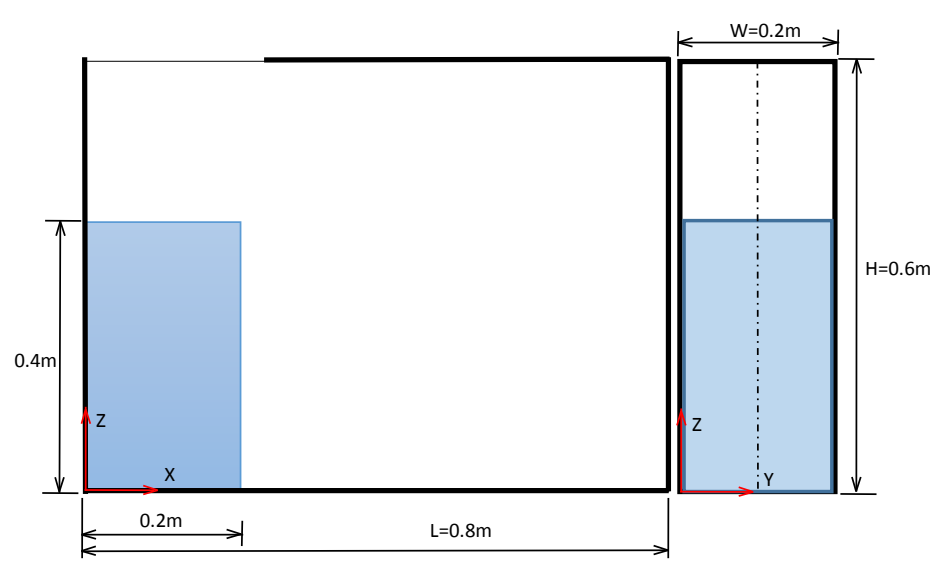

Fig. 1: A side view (left) and front view (right) of tank used with column water level

A Cartesian grid technique is used for the spatial discretization. The geometry domain size was similar with a previous experimental setup ${ }^{5)}$ as shown in Fig.1. Table 1 shows the three-dimensional modeling test matrix which include the total of nodes size, grid spacing and time step used in the simulation. For incompressible LBM flow, the value of time step $\Delta t$ is selected in order to keep the
Mach number low ${ }^{24)}$. A ratio of $\Delta t / \Delta x \approx 0.0025$ was used to achieve computational stability.

Table 1: Grid resolution studies

\begin{tabular}{|l|l|l|l|l|l|c|}
\hline $\begin{array}{l}\text { Grid } \\
\text { No. }\end{array}$ & $\mathbf{N x}$ & $\mathbf{N y}$ & $\mathbf{N z}$ & \#Nodes & $\Delta x$ & $\Delta t$ \\
\hline Grid 1 & 80 & 20 & 60 & 96000 & $\begin{array}{l}1.0 \\
\times 10^{-2}\end{array}$ & $2.5 \times 10^{-5}$ \\
\hline Grid 2 & 160 & 40 & 120 & 768000 & $\begin{array}{l}5.0 \\
\times 10^{-3}\end{array}$ & $\begin{array}{l}1.25 \\
\times 10^{-5}\end{array}$ \\
\hline Grid 3 & 320 & 80 & 240 & 6144000 & $\begin{array}{l}2.5 \\
\times 10^{-3}\end{array}$ & $\begin{array}{l}6.25 \\
\times 10^{-6}\end{array}$ \\
\hline
\end{tabular}

In order to test the computational performance of our cluster, we first checked the amount of speedup obtained by using Multi-Passage-Interface (MPI) parallel computing. Calculations from grid 1 to grid 3 were carried out on an Intel ${ }^{\circledR}$ Xeon ${ }^{\circledR}$ with 32 GB of RAM and 200GB memory size. The cluster platform with an Intel Xeon ®3.33 GHz (Processor X5680) has nodes with each node composes of two CPUs and each CPU comprises of six cores.

Fig. 2 plots the parallel computing performance for grid independent test. The speed up value is defined by $S(n)=T_{s n} / T_{n}$ which is defined as the ratio between time required by sequential run of simulation, $T_{s n}$ and the time required by a parallel run, $T_{n}$ using $n$ cores $^{24)}$. The figure shows that the speed up corresponding to simulations with 12 cores with fine grid is approximately 5 , and 9 cores corresponded to a speed up to 5.5. In general, the figure indicates the speed up of the MPI computing over sequential run is increased with the increase of the grid number. These results proved that our MPI parallel is able to achieve satisfactory speedup especially for large scale cases.

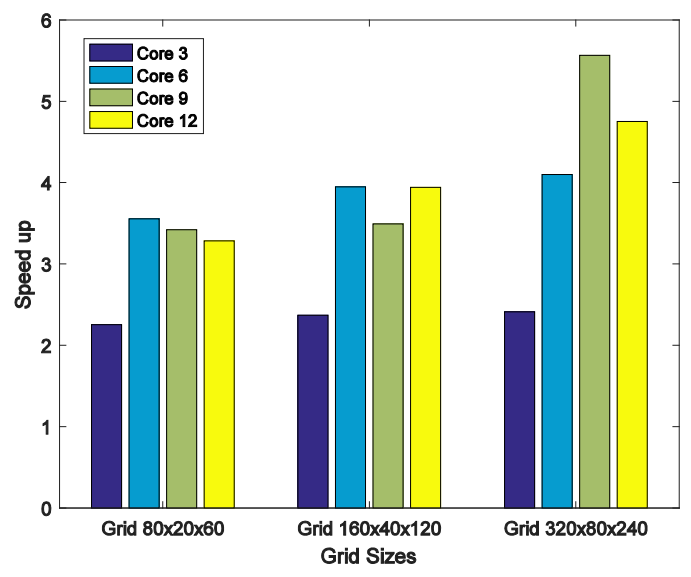

Fig. 2: MPI parallel computing performance for grid independent test 


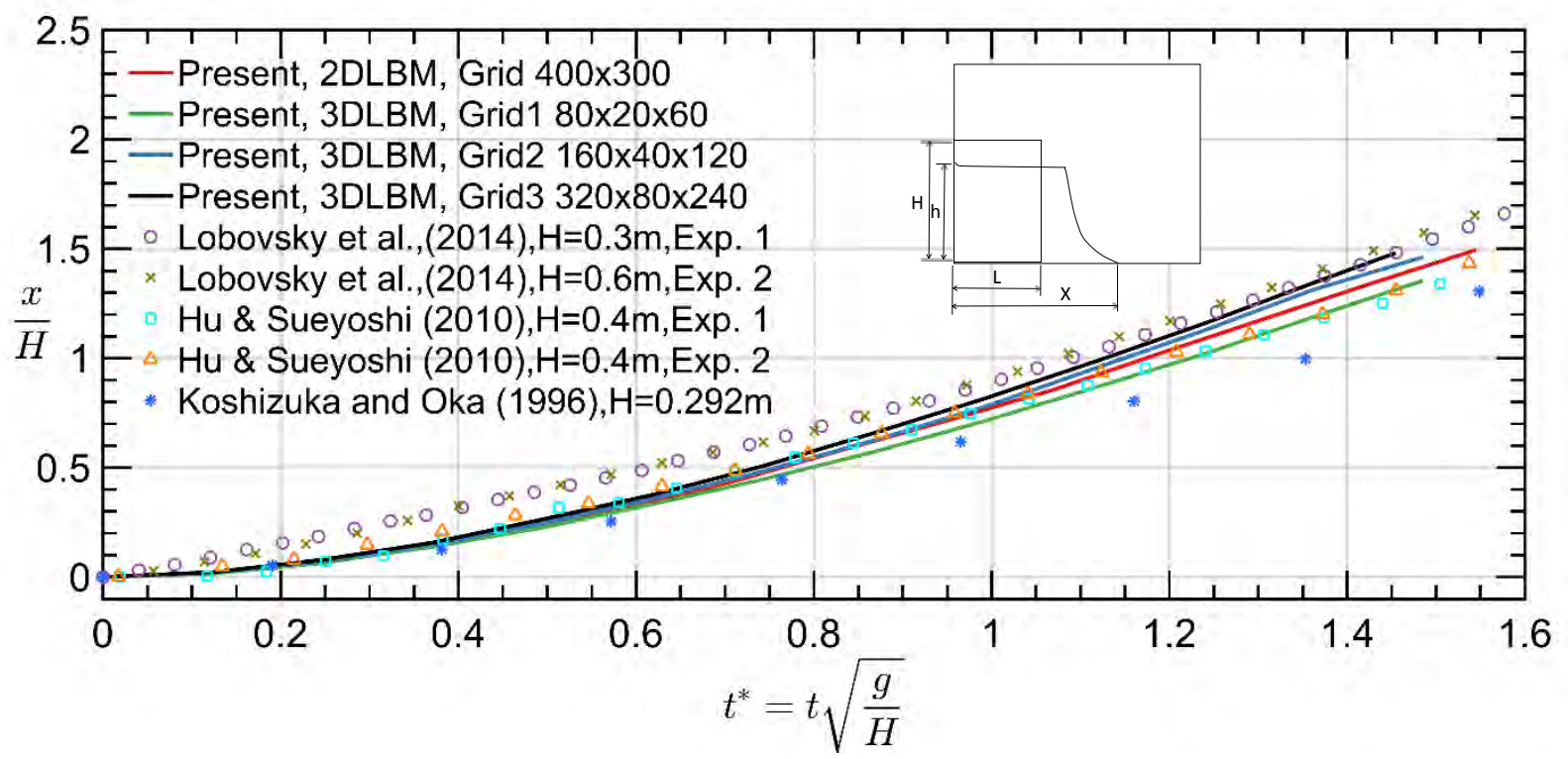

Fig.3: Comparison of numerical and experimental results for the surge front position with data from previous studies $3,5,25)$

\section{Results}

\subsection{Water front and free surface profile}

This study used well-established documented experimental techniques ${ }^{3,4,5,25)}$ to verify the numerical simulation. In Fig. 3, we expected the wave front speed to decrease as the mesh is refined since it corresponds to better satisfaction of the no-slip boundary condition. However, our computed results show an increase in the speed of the wave front. This may be attributed to nature of the wall boundary conditions employed in LBM i.e. bounce back boundary which would result in a different behavior with mesh refinement. On another point, the two-dimensional seems to provide a rather accurate and consistent behavior when compared with the three dimensional result taken at the mid-plane of the domain. In general, the computed solution agree well with a previous experimental data ${ }^{5)}$ however it is rather inconsistent with other previous data ${ }^{3,24)}$. This may be due to the results of using different liquid column dimensions (size) as well as different experimental setups, i.e. gate mechanism.

Fig. 4 depicts a comparison of the free surface profile between the experimental data, 2D LBM and 3D LBM taken at the mid-section of the domain. Aside from the gate effect at the early stages of the solution, the computed solutions agree well with experiment.

The computed solutions show the 3D effect of wave front to be faster when compared to the $2 \mathrm{D}$ solution. It was also found to be faster than the experimental data ${ }^{25)}$. This may be due to the friction that happened between fluid and bottom wall from the experiment ${ }^{25}$. This could be attributed to the wall effect from the side of the computational domain. An interesting way to confirm that is to repeat the computation for different domain sizes in the $y$-dim. We expected the results to resemble the 2D case as the domain gets wider. However, such study is beyond the scope of this work and thus left as future work.

\subsection{Three-dimensionality effect}

By improving the experimental setup from previous studies $^{5,8)}$ with a new gate removal mechanism system which is lighter and a new high-speed resolution camera, the effect of 3D can be clearly observed. The strong turbulent flow pattern with splash droplet effect also can be captured.

Fig. 5 and 6 shows the 3D free surface profile taken from side-view and perspective view. From a qualitative point of view, the computed results compare well with the experiment images. However, after a closer look it would seem that 3D LBM tend to over-estimate splash effect after impact and under-estimate maximum volume of water that rises over the wall after impact. This could be caused by the effect of the gate motion which is not considered in the current study. The initial shape of water front may be influenced by the efficiency of the gate motion and can be affected by the strong flow pattern after arriving at the impact area. The comprehensive wave impact study can be extended after this phenomenon is deeply investigated. 

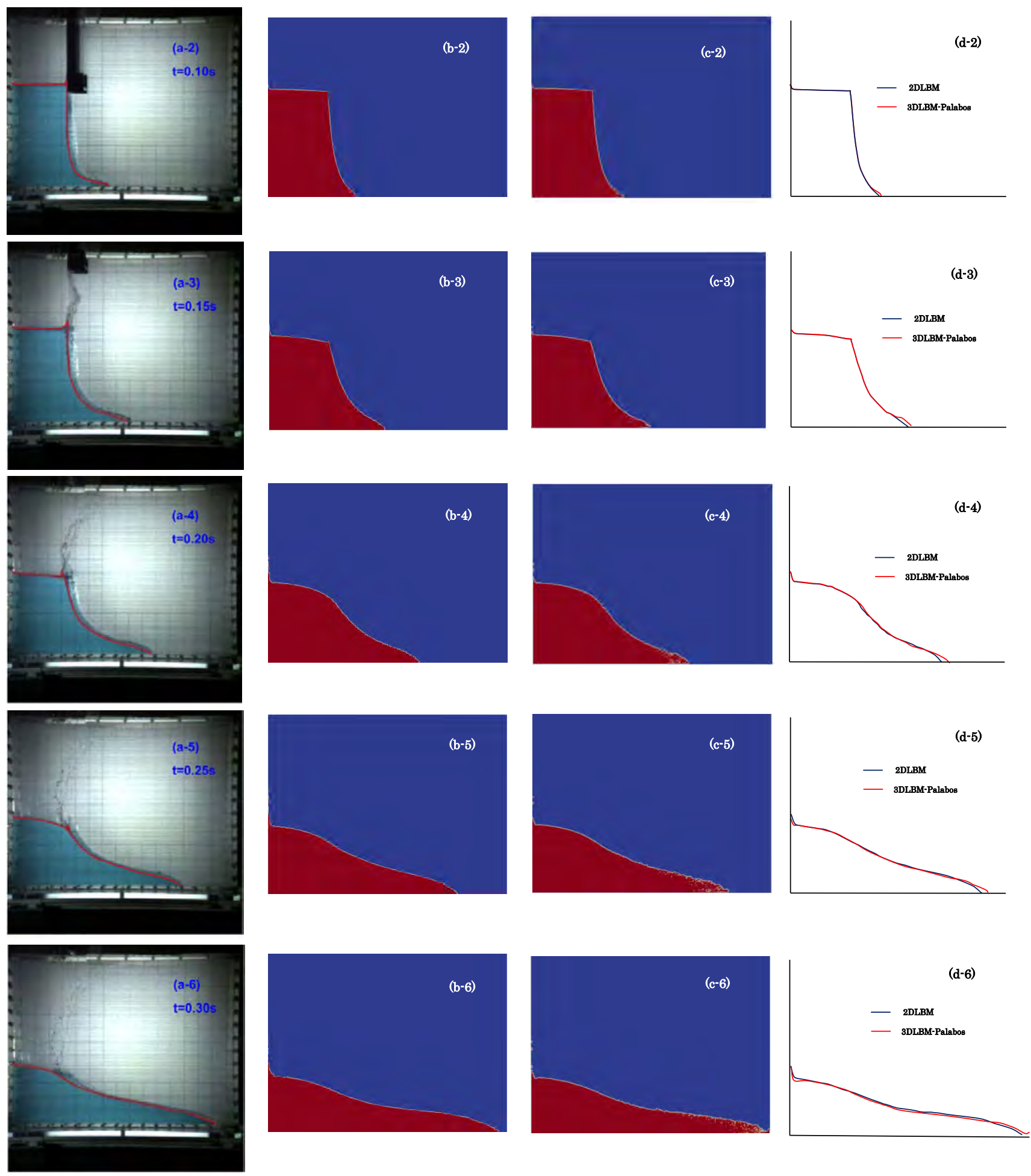

Fig. 4: Evolution of free surface profile, from top to bottom: a-1 to a-6 show the experimental data from literature ${ }^{9}$, $\mathbf{b}-\mathbf{1}$ to b-6 show the results for 2DLBM, c-1 to c-6 show the results for 3DLBM and $\mathbf{d - 1}$ to $\mathbf{d}-\mathbf{6}$ show the comparison between 2DLBM and 3DLBM 

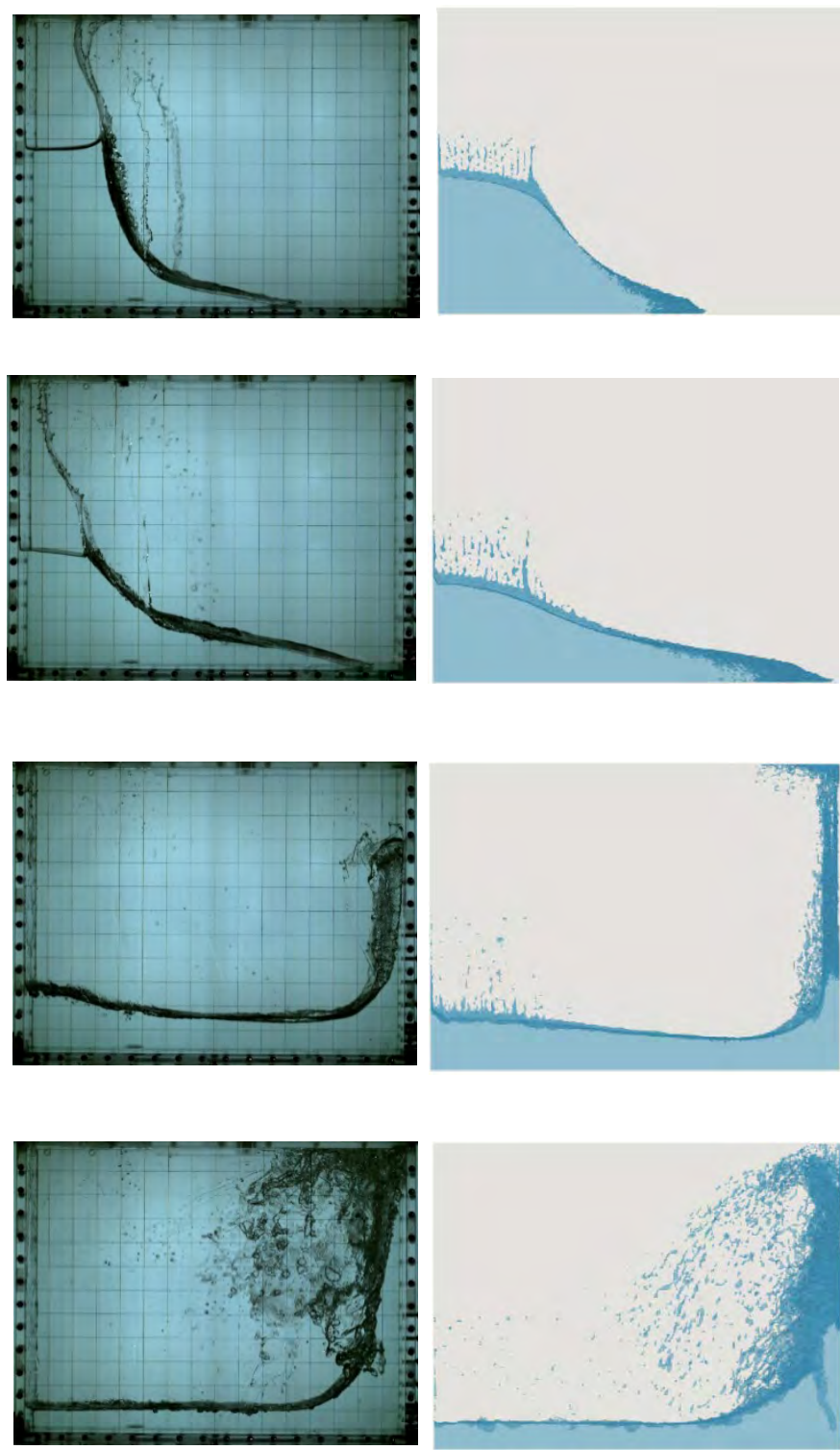

Fig.5 : From side view; comparison from experimental data and 3DLBM numerical solution for evolution of free surface profile at times 0.2, 0.29, 0.47 and $0.74 \mathrm{~s}$.

\section{Conclusion}

In this paper, a 3D free surface flow using free surface lattice Boltzmann Method (LBM) was developed. The 2D seems to provide a rather accurate and consistent behavior when compared with the $3 \mathrm{D}$ result and generally, the computed solution agree well with a previous experimental data ${ }^{5}$. Additionally, from a qualitative point of view, the computed results from the side and perspective view are consistent with the experimental data. In order to achieve stability, accuracy and reduce time of the numerical results, the turbulent Smagorinsky LES and MPI parallelism were implemented. In the future, the gate removal and pressure effect of $3 \mathrm{D}$ modeling should be investigated more thoroughly in order to consider the model's capability of the real-world fluid simulation.
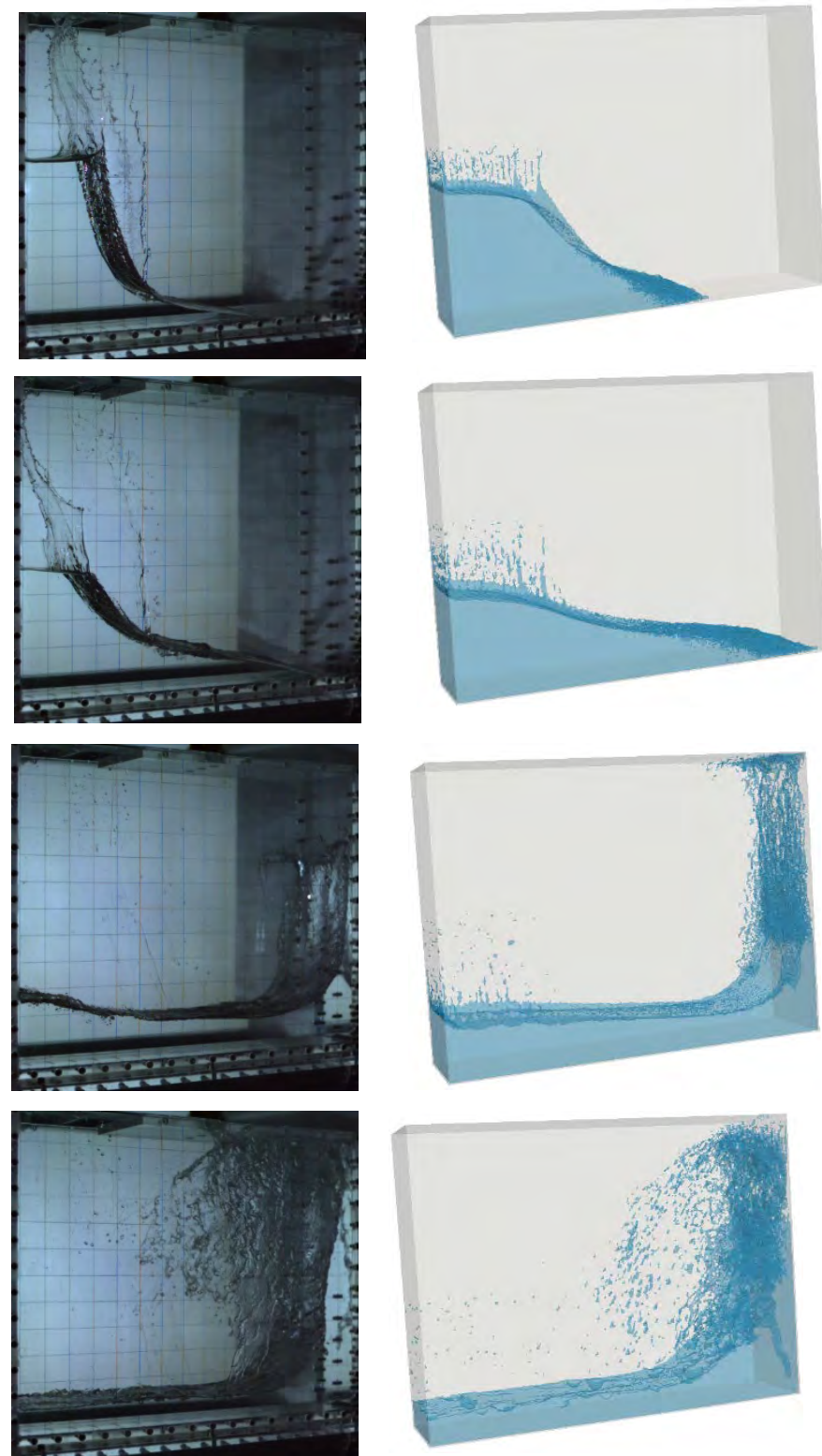

Fig.6 : From perspective view; comparison from experimental data and 3DLBM numerical solution for evolution of free surface profile at times 0.2 , $0.29,0.47$ and $0.74 \mathrm{~s}$.

\section{Acknowledgement}

The authors would like to thank Kyushu University for sponsoring the first author for a research internship at Karlsruhe Institute of Technology (KIT), Germany under the support of Oversea Research Internship Program 2016. This internship had contributed greatly towards the understanding of the application of LBM technique particularly in solving free surface problems. Special thanks go to Dr. Mathias Krause, J. from KIT . 


\section{References}

1) M. Brocchini and D.H.Peregrine. Advances in Coastal and Ocean Engineering. Volume 8. World Scientific Publishing Co.Pte.Ltd (2002)

2) Janßen C, Krafczyk M. Comput Math with Appl. 61(12):3549-3563 (2011).

3) Lobovský L, Botia-Vera E, Castellana F, Mas-Soler J, Souto-Iglesias A. J Fluids Struct.48:407-434 (2014)

4) Martin J, Moyce W. Philos Trans R Soc London Ser A, Math Phys Sci. 244(882):325-334 (1952)

5) $\mathrm{Hu} \mathrm{C}$, Sueyoshi M. Numerical simulation and experiment on dam break problem. J Mar Sci Appl. 9(2):109-114 (2010)

6) Lee T, Zhou Z, Cao Y. J Fluids Eng. 124(1):215-226. (2012)

7) Kleefsman KMT, Fekken G, Veldman AEP, Iwanowski B, Buchner B. J Comput Phys. 206(1):363-393 (2005)

8) Liao K, Hu C, Sueyoshi M. Appl Ocean Res. 50:192208 (2015)

9) Ye Z, Zhao X, Deng Z. J Mar Sci Technol. 21(4):579-591 (2016)

10) Janßen CF, Grilli ST, Krafczyk M. Comput Math with Appl. 65(2):211-229 (2013)

11) He X, Luo L-S. Phys Rev E. 56(6):6811-6817 (1997)

12) Shan $X$, Rothman S. flows. Phys Rev E. 47(3) (1993)

13) Jiang F, Hu C. Appl Ocean Res. 45:1-9 (2014)

14) Xuhui L. Doctoral Thesis, Kyushu University (2016)

15) http://www.palabos.org. Accessed October 1, 2016

16) Janssen C, Krafczyk M. Comput Math with Appl. 59(7):2215-2235 (2010)

17) Quian, Y. H., D’Humieres D, Lallemand P. Lattice BGK models for Navier Stokes equations. Europhys Lett. (17):479- 484 (1992)

18) Korner, $C$, Thies $M$, Hofmann, $T$, Thurey, $N$ and Rude U. J Stat Phys. 121:179-196 (2005)

19) Thurey N. Doctoral Thesis, University of ErlangenNuremberg, Germany (2007)

20) Thies M. Doctoral Thesis, University of ErlangenNuremberg, Germany (2005)

21) Manfred Krafczyk, Jonas Tolke L-SL. Int J Mod Phys B. 17:33-39 (2003)

22) Janßen C, Krafczyk M. Comput Math with Appl.61(12):3549-356 (2011)

23) Parmigiani A, Latt J, Begacem MBEN, Chopard B. Int J Mod Phys C. 24(12):1-8 (2013)

24) Thorimbert Y, Latt J, Cappietti L, Chopard B. Int J
Mar Energy. 14:41-51(2016)

25) Koshizuka S, Oka Y. Nucl Sci Eng.123(3):421-434 (1996) 